\title{
ROTATION RATE OF ACTIVE NESTS ON THE SUN
}

\author{
LIDIA VAN DRIEL-GESZTELYI ${ }^{1,2}$, ED B.J. VAN DER ZALM ${ }^{1}$ \\ and CORNELIS ZWAAN 1 \\ ${ }^{1}$ Astronomical Institute, University of Utrecht, Postbus 80.000 , \\ 3508 TA Utrecht, The Netherlands \\ 2 Kiso Observatory, Institute of Astronomy, University of Tokyo, \\ Mitake-mura, Kiso-gun, Nagano-ken, 397-01 Japan
}

\begin{abstract}
We analyse Greenwich sunpot group position data for one solar cycle (1952-1963) in order to study the rotation rate of clusters of sunspot groups, so-called active nests. We find that the latitude-dependent rotation rate of nests shows an intrinsic spread. This spread appears to be caused by extensive and dense clusters of sunspot nests (superclusters or nested nests), and by very compact single nests of short duration. The 'best' rotation rate is defined as the rate at which the highest number of sunspot groups appear to form nests. This latitude-dependent best rotation is close to the rotation rate of individual recurrent sunspots, although nests seem to rotate slightly more rigidly than sunspot groups.
\end{abstract}

\section{INTRODUCTION}

The probability of flux emergence is more than 10 times higher within existing active regions than in quiet parts of the activity belts (Ligget and Zirin 1985, Harvey and Zwaan 1993). At least $30 \%$ of sunspot groups are born in very small "nests", emerging within a few heliographical degrees from existing or recently decayed sunspots (see e.g. Gaizauskas et al., 1983; Brouwer and Zwaan 1990).

The rotation rates of nests are important in the study of large-scale patterns of solar activity. The considerable spread in rotation rates found earlier (van Driel-Gesztelyi et al., 1992a) prompted a refined analysis of nest rotation rates for one solar cycle (1952-1963).

The results of this analysis on a longer dataset will be published in more detail (van Driel-Gesztelyi et al., 1993).

\section{DEFINITION OF ACTIVE NESTS}

According to our definition of active nests, successive members have to appear in a distance smaller than $\left(\frac{\Delta L^{\prime}}{4^{\circ}}\right)^{2}+\left(\frac{\Delta \Phi}{2^{\circ}}\right)^{2} \leq 2$, where $\Delta L^{\prime}$ and $\Delta \Phi$ are the differences between the longitudes and latitudes of the sunspot groups. The difference in longitude $\Delta L^{\prime}$ is corrected for an adjustable differential rotation (v) with respect to the Carrington rotation, as: $\Delta L^{\prime}=\left|L_{i+1}-L_{i}-v\left(t_{i+1}-t_{i}\right)\right|$ with $v=a-b \sin ^{2} \Phi$ where $a$ and $b$ are 


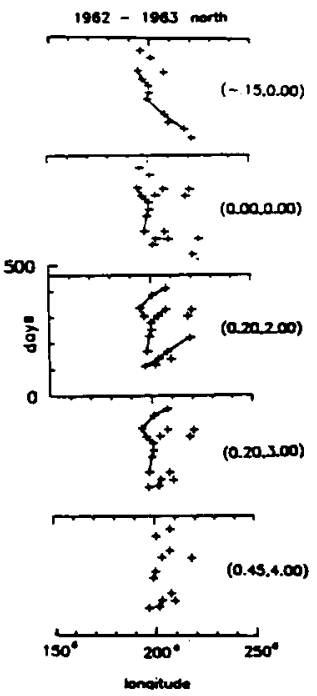

FIGURE 1. An example of a cluster of nests $\left(L \approx 200^{\circ}, \Phi \approx 12^{\circ}\right.$ ) shown with different rotation parameter combinations. In this supercluster, sunspots kept appearing for one year close to each other in space and time. As a result, smaller sub-nests can be found at any rotation rate (shown by unconnected crosses) and even big nests ( $M \geq 4$ - members are connected) with a wide variety of rotation parameter combinations. The highest number of sunspot groups (14) belonging to big nests was found with parameters $a=0.2$ and $b=2.0$.

TABLE I. Percentage of sunspot groups $(\Sigma=958)$ contained in clusters of at least 4 members as function of rotation parameters $a$ and $b$ for the period of 1957-1959 on the Northern hemisphere of the Sun.

a./ Real data

\begin{tabular}{|c|c|c|c|c|c|c|c|c|c|c|c|c|c|}
\hline$b$ & \multicolumn{13}{|c|}{$a$} \\
\hline & -0.15 & -0.10 & -0.05 & 0.00 & 0.05 & 0.10 & 0.15 & 0.20 & 0.25 & 0.30 & 0.35 & 0.40 & 0.45 \\
\hline 0.0 & 14 & 12 & 11 & 12 & 11 & 12 & 12 & 14 & 11 & 8 & 6 & 6 & 8 \\
\hline 0.5 & 12 & 13 & 10 & 12 & 12 & 12 & 13 & 11 & 11 & 11 & 9 & 7 & 7 \\
\hline 1.0 & 13 & 10 & 11 & 10 & 10 & 14 & 13 & 11 & 12 & 10 & 11 & 8 & 6 \\
\hline 1.5 & 10 & 13 & 11 & 10 & 12 & 11 & 15 & 14 & 11 & 11 & 11 & 9 & 7 \\
\hline 2.0 & 12 & 12 & 10 & 10 & 11 & 10 & 13 & 16 & 12 & 11 & 10 & 10 & 7 \\
\hline 2.5 & 11 & 12 & 137 & 10 & 10 & 11 & $\overline{12}$ & 13 & 11 & 11 & 12 & 12 & 10 \\
\hline 3.0 & 8 & 12 & 11 & 12 & 10 & 9 & 11 & 16 & 14 & 11 & 12 & 11 & 9 \\
\hline 3.5 & 9 & 9 & 11 & 12 & 12 & 10 & 10 & 14 & 14 & 13 & 11 & 10 & 10 \\
\hline 4.0 & 8 & 9 & 11 & 11 & 12 & 12 & 10 & 12 & 15 & 13 & 11 & 12 & 11 \\
\hline 4.5 & 6 & 8 & 10 & 9 & 11 & 11 & 10 & 10 & 13 & 14 & 11 & 12 & 12 \\
\hline 5.0 & 6 & 9 & 8 & 9 & 10 & 9 & 11 & 10 & 12 & 14 & 11 & 10 & 12 \\
\hline
\end{tabular}

b./ Average values of five datasets with randomized longitudes of sunspot groups.

\begin{tabular}{l|lllllllllllll}
\hline 0.0 & 6 & 6 & 6 & 6 & 6 & 6 & 5 & 6 & 6 & 6 & 6 & 5 & 6 \\
0.5 & 5 & 7 & 6 & 6 & 6 & 6 & 6 & 6 & 6 & 6 & 5 & 6 & 6 \\
6.0 & 6 & 6 & 7 & 6 & 6 & 6 & 6 & 5 & 5 & 6 & 5 & 6 & 6 \\
6.5 & 6 & 6 & 6 & 7 & 6 & 7 & 7 & 5 & 5 & 5 & 6 & 6 & 6 \\
2.0 & 5 & 6 & 5 & 6 & 6 & 6 & 6 & 6 & 5 & 5 & 5 & 6 & 6 \\
2.5 & 5 & 6 & 6 & 7 & 6 & 6 & 6 & 6 & 6 & 6 & 5 & 5 & 5 \\
3.0 & 6 & 6 & 6 & 6 & 6 & 6 & 6 & 6 & 6 & 5 & 6 & 5 & 6 \\
3.5 & 6 & 6 & 6 & 6 & 6 & 6 & 6 & 7 & 6 & 6 & 6 & 6 & 5 \\
4.0 & 6 & 6 & 6 & 5 & 6 & 6 & 6 & 6 & 7 & 6 & 5 & 5 & 6 \\
4.5 & 7 & 7 & 6 & 6 & 6 & 6 & 6 & 6 & 6 & 6 & 6 & 6 & 6 \\
5.0 & 6 & 6 & 6 & 6 & 5 & 7 & 6 & 6 & 6 & 6 & 6 & 6 & 6 \\
\hline \hline
\end{tabular}


adjustable rotation parameters; and $\Phi=\frac{\left(\Phi_{i+1}+\Phi_{i}\right)}{2}$. Concerning the succession in time, some maximum time gap $\tau \leq 72^{\mathrm{d}}$ is allowed between the successive sunspot group appearances in a nest.

\section{ROTATION RATE OF SUNSPOT NESTS}

In order to find the characteristic rotation parameters $(a, b)$ we counted sunspot groups in nests with at least 4 members $(M \geq 4)$ over a wide range of $(a, b)$ parameters. As an example, results for the period 1957-1959 are shown in Table Ia as a percentage of the total of 958 sunspot groups occurring during that period. The same analysis applied to 5 samples of data with randomized longitudes (based on the same dataset) gives the average values shown in Table $\mathrm{Ib}$. A comparison between Table Ia and $\mathrm{Ib}$ shows that active nests are real, and not accidental formations.

The highest numbers of sunspot groups belonging to large nests are situated along a diagonal bar in the matrix, with a maximum around $(a=0.2, b=2.0-3.0)$ in Table Ia, which can therefore be regarded as the 'best' rotation rate of nests in that period, while in Table $\mathrm{Ib}$ the numbers are quite uniform and much lower than in Table Ia. For the rotation of single, recurrent sunspots Newton and Nunn (1951) found ( $a=0.19, b=2.69)$, close to the 'best' rotation rate of Table Ia. Note, that for slightly different rotation parameters our procedure still selects nearly the same sunspot groups for the same nests, only for quite different rotation parameters does the program cluster sunspot groups in different ways (see Fig. 1). The diagonal bar of relatively high percentages in Table Ia contains sets of rotation parameters which correspond to nearly the same rotation velocity in the middle of the sunspot zone.

In the real data (Table Ia) we find substantially more nests than in the randomized data (Table $\mathrm{Ib}$ ) for quite a range of rotation parameters. One cause is the occurrence of superclusters: relatively dense clusters of nests (see Fig. 1), in which individual nests show up for a wide range of rotation rates, the clustering of sunspot groups depending sometimes on the rotation parameter sets. Another cause for the large spread in possible parameter sets is the occurrence of shortlived but compact nests, in which the members are found to be clustered for a large range of rotation rates.

Despite the intrinsic spread, a 'best' rotation rate of nests can be defined: this is the rate at which the highest number of sunspot groups appear to form nests. However, this 'best' rate is somewhat arbitrary and it seems to change somewhat with time. In any case, this rate is close to the rotation rate of individual recurrent sunspot groups, although nests seem to rotate slightly more rigidly than sunspot groups.

\section{REFERENCES}

Brouwer, M.P., Zwaan, C.: 1990, Solar Phys. 129, 221.

Gaizauskas, V., Harvey, K.L., Harvey, J.W. and Zwaan, C.: 1983, Astrophys. J. 265, 1056.

Harvey, K.L., and Zwaan, C.: 1993, submitted to Solar Phys.

Ligget, M., and Zirin, H.: 1985, Solar Phys. 97, 51 .

Newton, H.W., and Nunn, M.L. 1951 Monthly Notices Roy. Astr. Soc. 111, 413.

van Driel-Gesztelyi, L., van der Zalm, E.B.J. and C. Zwaan.: 1992, in K.J. Harvey (ed.) The Solar Cycle A.S.P. Conf. Ser. 27, 89.

van Driel-Gesztelyi, L., van der Zalm, E.B.J., Zwaan, C.: 1993, in preparation 